\title{
Impact du décorticage du tournesol sur la valeur nutritionnelle et l'intérêt économique des tourteaux en fabrication d'aliments composés
}

\author{
Corinne PEYRONNET ${ }^{1}$ \\ Frédéric PRESSENDA ${ }^{2}$ \\ Alain QUINSAC ${ }^{3}$ \\ Patrick CARRÉ 4 \\ 1 ONIDOL, \\ 11, rue de Monceau, \\ CS 60003, \\ 75378 Paris cedex 08 \\ $<$ c.peyronnet@onidol.fr> \\ ${ }^{2}$ CEREOPA, \\ 16, rue Claude Bernard, \\ 75231 Paris cedex 05 \\ ${ }^{3}$ CETIOM, \\ rue Monge, \\ Parc Industriel, \\ 33600 Pessac \\ 4 CREOL, \\ rue Monge, \\ Parc Industriel, \\ 33600 Pessac
}

Article reçu le 24 septembre 2012

Accepté le 2 octobre 2012

La dépendance française et plus encore européenne en protéines végétales pour le secteur de l'alimentation animale incite à la recherche de nouvelles sources de protéines alternatives pouvant se substituer aux protéines d'importation telles que le tourteau de soja.

La France dispose de sources de protéines variées et notamment des tourteaux de colza et tournesol issus de graines produites en France et dont la consommation totale sur la campagne 2010/2011 dépassait largement les 3 millions de tonnes comparées aux 3,8 millions de

\begin{abstract}
Effect of dehulling on nutritional value and economical interest of sunflower meal for feedstuff

Sunflower meal is an alternative source of proteins for feedstuff but its high fiber content reduces the nutritional value. Different dehulling processes can improve the protein content and the nutritional value of meal. Partially dehulled sunflower meals with $32 \%$ and $36 \%$ of proteins produced in different crushing plants in France had been compared to non-dehulled sunflower meal (29\% of proteins). The total French feedstuff production was simulated with a model including prices of raw materials observed during 20102011 campaign. The interest prices of the different qualities of sunflower meal compared to soybean meal varied from $0.43,0.50$ to 0.70 for 29,32 and $36 \%$ of proteins content respectively. The $36 \%$ protein sunflower meal seems very well adapted to laying hens and ducks diets where 29\% type should be more adapted to meat cows, sows and rabbits who need less concentrated diets. The new market for the $36 \%$ protein sunflower meal type could notably help to provide local sources of proteins for high quality poultry production.
\end{abstract}

Key words: sunflower, meal, dehulling, feedstuff, economic interest

tonnes de tourteau de soja importé d'Amérique du Sud et des États-Unis.

Ces sources de protéines métropolitaines sont modérément concentrées, avec de moindres teneurs en protéines et de plus fortes teneurs en cellulose que le tourteau de soja lui-même très concentré et par conséquent très polyvalent. Les traitements technologiques appliqués et notamment le décorticage préalable des graines avant la trituration ont un impact sur les caractéristiques de composition des tourteaux et influent directement sur les valeurs nutritionnelles et les taux d'incorporation potentiels dans les différentes formules d'aliments composés. Ces procédés sont par conséquent déterminants pour l'intérêt économique des tourteaux.

Cet article présentera la technique du décorticage et ses effets sur les graines de tournesol et sur les caractéristiques chimiques et nutritionnelles des tourteaux. L'optimisation du taux de décorticage pour l'amélioration de la qualité des tourteaux et la valorisation des coques produites sera abordée dans la perspective de l'application du

Pour citer cet article : Peyronnet C, Pressenda F, Quinsac A, Carré P. Impact du décorticage du tournesol sur la valeur nutritionnelle et l'intérêt économique des tourteaux en fabrication d'aliments composés. OCL $2012 ; 19$ (6) : 341-346. doi : 10.1684/ocl.2012.0486 
décorticage dans une unité industrielle de grande capacité équipée d'une chaudière à combustion de biomasse. L'intérêt économique des tourteaux produits sera également étudié à l'échelle du secteur des aliments composés français.

\section{Qualité des tourteaux de tournesol décortiqués}

\section{Caractéristiques des graines, amandes et coques}

Les proportions de coques des graines de tournesol sont comprises entre 22 et $28 \%$ et varient en fonction du cultivar, de la taille des graines et de leur teneur en huile car l'amélioration de la teneur en huile des variétés par sélection s'est faite au détriment de la teneur en coques et de l'aptitude des graines au décorticage. Les amandes sont composées de plus de $80 \%$ d'huile et de protéines, ce qui permettrait d'obtenir, dans I'hypothèse d'un déshuilage complet, un tourteau de teneur en protéines supérieure à $50 \%$ (tableau 1).

Les coques de tournesol contiennent seulement 2 à $3 \%$ de I'huile et $10 \%$ des protéines de la graine. En revanche, elles renferment l'essentiel des composés pariétaux : $85 \%$ de la fraction NDF (soluble dans un détergent neutre), $90 \%$ de la fraction ADF (soluble dans un détergent acide) et $95 \%$ de la fraction ADL (lignine) contenues dans la graine. Le décorticage est donc un bon moyen de séparer les composés pariétaux sans perte importante de protéines et d'huile. L'aptitude au décorticage est déterminée génétiquement et pourrait être améliorée par la sélection. Ce caractère dépend de la structure du sclérenchyme de la coque et de la proportion de massifs de cellules faiblement ou fortement lignifiées (figure 1). Ces dernières favorisent la rigidité de la coque et sa rupture sous l'effet des contraintes mécaniques dans le décortiqueur (Beauguillaume, 1982).

\section{Les procédés de décorticage}

Plusieurs types de décortiqueurs existent et se distinguent par la manière dont des chocs sont appliqués aux graines. Le type "Bühler » consiste à propulser les graines par la force centrifuge contre une paroi. Les inconvénients sont la production de fines et le transfert de I'huile dans les
Tableau 1. Composition moyenne des graines, amandes, coques et tourteaux de tournesol. D'après (Carré, 2010).

\begin{tabular}{|llllll|}
\hline & Graines & Amandes & Coques & $\begin{array}{l}\text { Tourteau } \\
\text { de graines non } \\
\text { décortiquées }\end{array}$ & $\begin{array}{l}\text { Tourteau } \\
\text { d'amandes }\end{array}$ \\
\hline $\begin{array}{l}\text { Matière sèche } \\
\text { (MS) \% }\end{array}$ & 92,8 & 90,5 & & 88,8 & 90,5 \\
\hline Huile (\%MS) & 48,0 & 61,3 & 2,5 & 2,2 & 1,2 \\
\hline Protéines (\%MS) & 16,7 & 20,6 & 6,2 & 31,9 & 52,6 \\
\hline $\begin{array}{l}\text { Cellulose brute } \\
\text { (\%MS) }\end{array}$ & 17,3 & 2,4 & 57,6 & 28,1 & 6,2 \\
\hline $\begin{array}{l}\text { Cendres brutes } \\
(\% M S)\end{array}$ & 3,5 & 3,6 & 3,2 & 7,1 & 9,2 \\
\hline NDF (\%MS) & 26,6 & 5,4 & 83,9 & 45,1 & 13,7 \\
\hline ADF (\%MS) & 19,5 & 2,7 & 64,9 & 32,0 & 7,0 \\
\hline ADL (\%MS) & 6,3 & 0,4 & 22,3 & 10,5 & 0,9 \\
\hline
\end{tabular}

coques, conséquences des vitesses de rotation élevées nécessaires. Avec les décortiqueurs de type compressiondécompression, la brusque décompression et expansion de l'air situé entre l'amande et la coque provoque leur séparation. Ce procédé couteux en énergie ne convient pas aux graines riches en huile et il est en pratique limité aux graines de tournesol de bouche pour la consommation humaine. Les décortiqueurs les plus performants sont de type multichocs et désignés sous le nom de décortiqueurs russes. Leur principe de fonctionnement est similaire à celui des batteurs équipant les machines moissonnant les céréales. Par rapport aux appareils " mono-choc » ils génèrent des impacts multiples d'intensité modérée, ce qui améliore le rendement du décorticage et réduit la formation de fines. Une version du décortiqueur multichocs est le "ripple-Mill » présenté figure 2.

La séparation des coques et des amandes est réalisée sur la base de la différence de leurs densités dans un courant d'air, les fines étant préalablement séparées par des tamis. Le coût de cette opération est

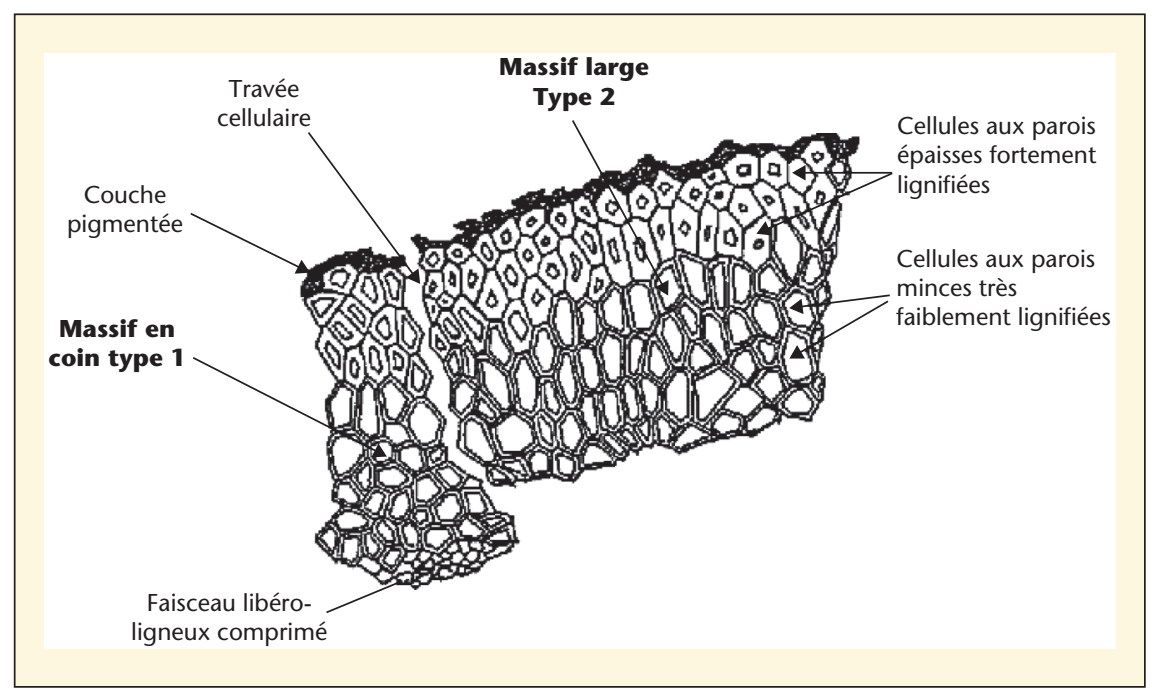

Figure 1. Structure des coques de tournesol (Beauguillaume, 1982). La figure montre de gauche à droite: un amas de cellules formant un massif en coin de type 1, une travée cellulaire séparant les deux amas, un amas de cellules formant un massif large de type 2. Les massifs de type 1 sont plus rigides que les massifs de type 2 et leur abondance favorise l'aptitude au décorticage. 


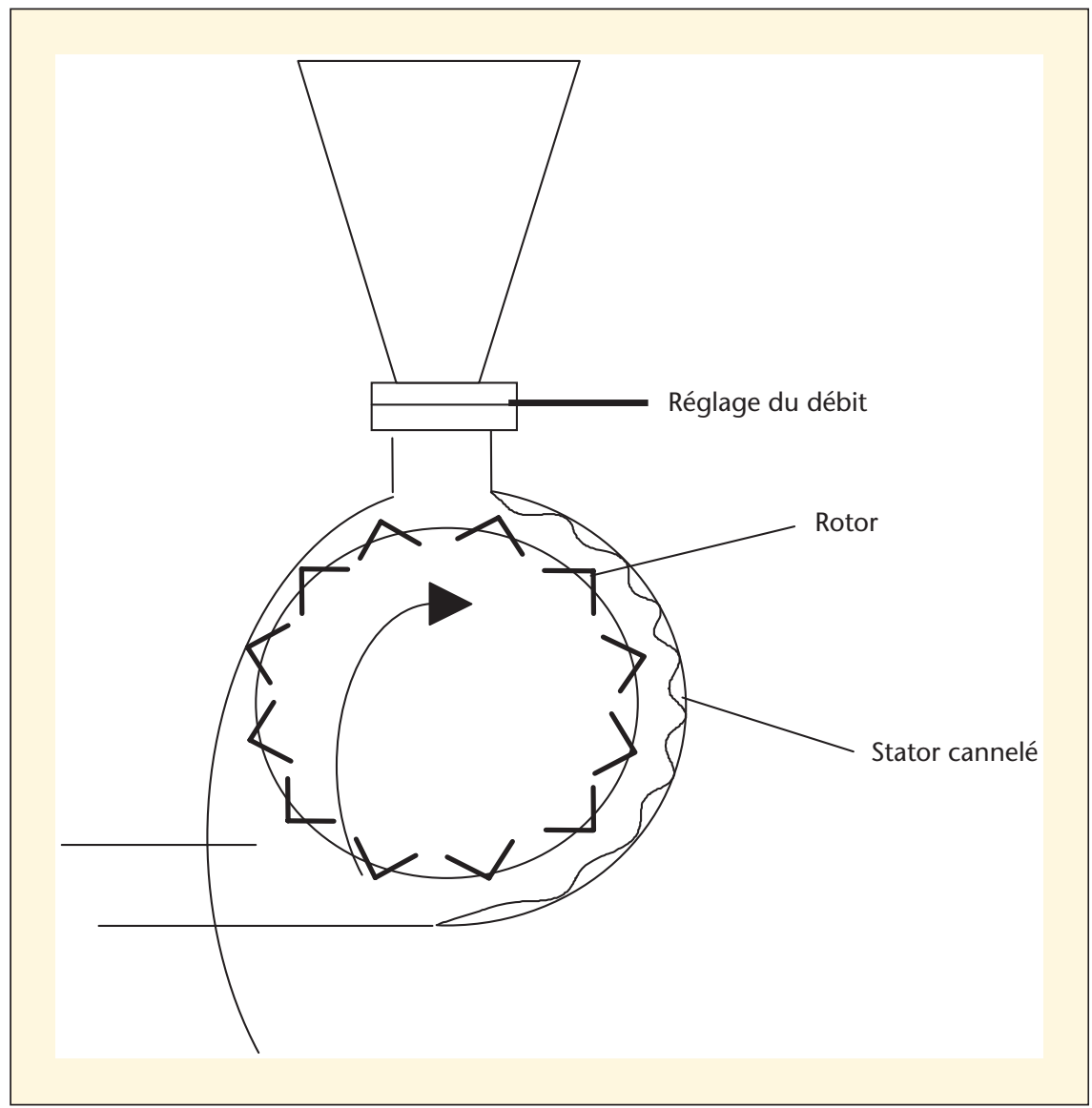

Figure 2. Décortiqueur multichocs type "Ripple mill ».

fortement impacté par la performance du décorticage et sa capacité à éviter la formation des fines. Des procédés utilisant les forces électrostatiques peuvent aussi être utilisés et ont l'avantage d'une moindre consommation en énergie (Sket-Cimbria, 1991).

\section{Contraintes et opportunités du décorticage du tournesol}

Du fait de son effet très positif sur la qualité des tourteaux de tournesol, le décorticage est devenu une pratique courante. II est cependant limité par deux facteurs: la valorisation parfois difficile des coques et la perte d'huile dans ces coques. Généralement, les coques sont valorisées par combustion pour générer l'énergie calorifique nécessaire à l'usine. Environ $60 \mathrm{~kg}$ de coques sont nécessaires pour fournir l'énergie calorique de la trituration d'une tonne de graines. Cette quantité représente $6 \%$ des graines et si celles-ci renferment $24 \%$ de coques, soit quatre fois plus, le taux de décorticage doit et ces valeurs sont obtenues en pratiquant un décorticage de $34 \%$. Des teneurs supérieures en protéines proches de $40 \%$ sont possibles. Elles supposent un taux de décorticage de $66 \%$, raisonnablement atteignable avec des décortiqueurs multichocs. Dans I'hypothèse d'une teneur en coques de $25 \%$, la quantité de coques séparée est d'environ $165 \mathrm{~kg} / \mathrm{t}$ ce qui, comme on vient de le voir, est théoriquement très supérieur aux besoins stricts de l'usine mais peut être néanmoins valorisé correctement. Le contexte actuel d'économies d'énergie d'origine fossile et le besoin de tourteau riche en protéines substituable au tourteau de soja concourent au développement du décorticage poussé, en usine de trituration.

\section{Développement de la production de tourteaux de tournesol décortiqués}

Les tourteaux partiellement décortiqués ont été développés en Argentine et se sont généralisés dans le monde mais restent minoritaires en France et ne sont actuellement produits que sur un site. Cependant, la mise en place d'une nouvelle unité de décorticage de graines de tournesol sur le site de Bassens (33) va permettre de produire un tourteau de tournesol à haute teneur en protéines et dont les caractéristiques devraient être de $36 \%$ de protéines et $19 \%$ de cellulose (par rapport à la matière brute) ou encore " type 36 ".

Le secteur de I'alimentation animale distingue classiquement deux types de tourteaux de tournesol utilisés en France, ceux issus de graines entières dénommés "tourteau de tournesol non décortiqué " ou encore "pailleux" et ceux issus de graines partiellement décortiquées dénommés "tourteau de tournesol partiellement décortiqué» comme indiqué dans les tables de composition et de valeur nutritive des matières premières (INRA-AFZ, 2004). Cependant, les formulateurs ont leurs propres matrices mises à jour par des analyses régulières des matières premières métropolitaines et d'importation disponibles sur le marché et disposent de données plus précises que celles des tables INRA-AFZ.

Les principaux critères de composition et valeurs nutritives issus de la banque 
de données 107 sont indiqués dans le tableau 2 pour les deux types de tourteaux et renseignent également sur la variabilité des deux paramètres déterminants que sont les teneurs en protéines brutes et en cellulose brute. Ainsi, il apparaît que les teneurs en protéines brutes sont proches de $28 \%$ pour les tourteaux non décortiqués et de $33 \%$ pour les tourteaux partiellement décortiqués. Cependant, les écarts types sont importants, conséquence de I'hétérogénéité des échantillons pris en compte mais également de la variabilité de composition et d'aptitude au décorticage des graines de départ (Dauguet et al., 2012) et du taux de décorticage appliqué. En effet, celui-ci peut varier largement comme il est développé dans l'article de Tostain et al. (2012) qui évoque l'équilibre à trouver entre taux de décorticage, combustion optimale des coques et qualité du tourteau pour une rentabilité économique globale de l'unité. Ainsi les écarts types des teneurs en protéines mentionnés dans ces tables et mesurés sur de très grands nombres d'échantillons sont de 2,2, du même ordre de grandeur que ceux de la cellulose brute. Même s'il existe une bonne relation entre ces deux critères, comme l'indique la figure 3, pour une teneur en protéines de $36 \%$ (soit près de $40 \%$ exprimés sur la matière sèche), la teneur en cellulose brute peut varier entre 16 et $21 \%$. Une telle variabilité est un facteur limitant pour les fabricants $d^{\prime}$ aliments du bétail qui doivent formuler au plus près des valeurs réelles pour éviter les marges de sécurité importantes et coûteuses.

Quant aux valeurs nutritionnelles des différentes qualités de tourteaux, cellesci sont assez mal renseignées pour les tourteaux partiellement décortiqués du fait du très faible nombre de mesures réalisées in vivo sur les tourteaux de tournesol français ces dernières années.

Cependant, Villamide et al. (1998), ont montré en 1998 que la teneur en énergie métabolisable (EMn) mesurée sur coqs étaient hautement corrélée $(P<0,001)$ aux constituants pariétaux et notamment à I'hémicellulose $(r=$ $-0,90)$, à l'ADL $(r=-0,84)$, au NDF $(r=-0,82)$ et également aux protéines brutes $(r=0,77)$.

Concernant les porcs, les travaux de Perez et al. (1986) réalisés sur 8 échantillons de tourteaux de tournesol
Tableau 2. Composition et valeur nutritionnelle des tourteaux de tournesol (INRA-AFZ, 2004) (valeurs exprimées par rapport au produit brut).

\begin{tabular}{|c|c|c|c|c|}
\hline & \multicolumn{2}{|c|}{$\begin{array}{l}\text { Tourteau partiellement } \\
\text { décortiqué } \\
\mathrm{N}=1141\end{array}$} & \multicolumn{2}{|c|}{$\begin{array}{l}\text { Tourteau non } \\
\text { décortiqué } \\
\mathbf{N}=2729 \\
\end{array}$} \\
\hline & Moy. & ET & Moy. & ET \\
\hline Matière sèche (\%) & 89,7 & 1,2 & 88,7 & 1,4 \\
\hline Protéines brutes (\%) & 33,4 & 2,2 & 27,7 & 2,2 \\
\hline Cellulose brute (\%) & 21,2 & 2 & 25,5 & 2,6 \\
\hline Matières grasses brutes (\%) & 1,7 & 0,6 & 2 & 0,8 \\
\hline Cendres brutes (\%) & 6,7 & 0,5 & 6,2 & 0,6 \\
\hline ED porc croissance $(\mathrm{kcal} / \mathrm{kg})$ & 2440 & & 2140 & \\
\hline EN porc croissance $(\mathrm{kcal} / \mathrm{kg})$ & 2220 & & 1090 & \\
\hline EMAn coq (kcal/kg) & 1500 & & 1350 & \\
\hline ED lapin (kcal/kg) & 2490 & & 2240 & \\
\hline UFL (par kg) & 0,66 & & 0,56 & \\
\hline UFV & 0,57 & & 0,46 & \\
\hline PDIN (g/kg) & 219 & & 178 & \\
\hline PDIE (g/kg) & 115 & & 93 & \\
\hline
\end{tabular}

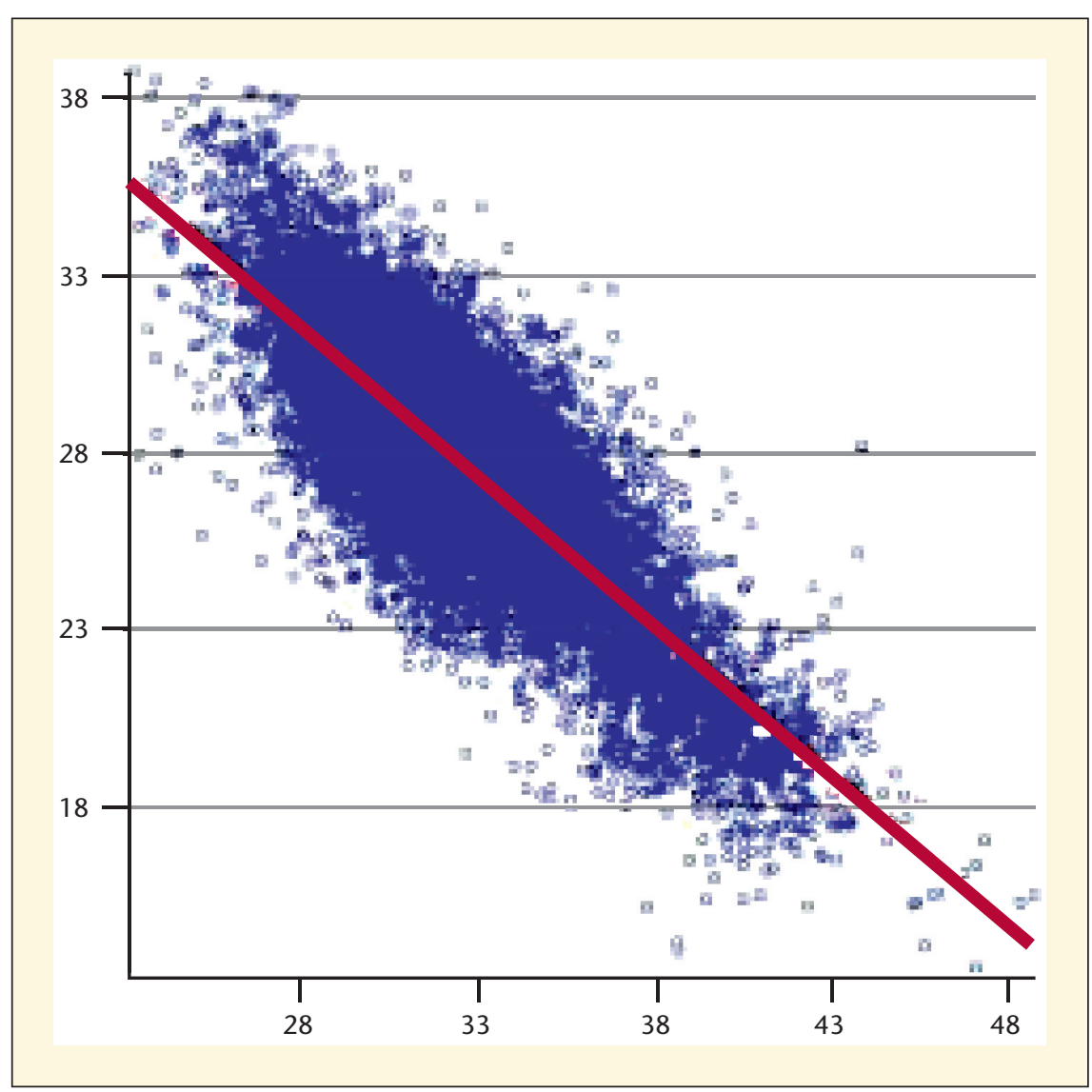

Figure 3. Relation entre teneurs en cellulose brute et protéines du tourteau de tournesol. $R 2=0,65$ ETR $=1,95 \quad P<0,01 \quad N=14171$. FeedBase.com. Cellulose brute (\% sur MS) = $55,21-0,84^{*}($ MAT \% sur MS). 
allant du tourteau d'amandes "type 40 " à un tourteau pailleux, ont montré que la valeur énergétique digestible (ED) dépend étroitement $(r=-0,96)$ de leurs teneurs en constituants pariétaux: cellulose brute (CB), ADF, NDF ou ADL. Du fait de la forte corrélation entre les différents constituants pariétaux, tous ces critères conduisent à des équations de prédiction de I'ED dont la précision est très voisine. La prise en compte du critère $C B$ très couramment analysé a donc été retenue et conduit à la relation linéaire suivante :

$\mathrm{ED}(\mathrm{kcal} / \mathrm{kg} \mathrm{MS})=4690-79 \mathrm{CB}(\% \mathrm{MS})$ avec $r=-0,958, \mathrm{Sr}=255$ et $\mathrm{CVr}=8 \%$

Cette équation demeure cependant imprécise puisque la variation résiduelle s'élève à $8 \%$. Une équation curvilinéaire dans laquelle la $C B$ explique à elle seule $97 \%$ de la variation a ainsi été établie.

Pour l'utilisation de la fraction azotée, l'équation suivante a été établie :

CUDN $=86,7-0,32(+/-0,05)$ CB \% MS avec $r=-0,93, \mathrm{Sr}=1,34$ et $\mathrm{CVr}=1,7 \%$.

Ces travaux, qui proposent des moyens simples d'estimation des valeurs nutritionnelles en fonction des teneurs en $C B$, mériteraient d'être mis à jour. Les variétés de tournesol actuelles, les conditions de décorticage ou encore les paramètres de trituration sont autant de facteurs à étudier pour préciser les valeurs nutritionnelles des différents tourteaux.

\section{Intérêt économique du tourteau décortiqué "type 36 "}

Un travail de simulation de l'intérêt économique d'un tourteau de tournesol 36 à l'échelle de la France entière a été réalisé par le CEREOPA à l'aide du modèle Prospective Aliment. Celui-ci simule I'incorporation des matières premières pour le secteur des aliments composés industriels français qui représentait en 2010/2011 plus de 19 millions de tonnes d'aliments répartis ainsi : $21 \%$ pour les bovins, $29 \%$ pour les porcs, $44 \%$ pour les volailles et $5 \%$ pour les autres animaux d'élevage. Le modèle prend également en compte la particularité des différentes régions en appliquant des coûts de transport spécifiques ce qui permet de tester l'intérêt des matières premières sur les différents bassins de productions animales.

Les simulations ont été réalisées avec des hypothèses de prix des matières premières correspondant aux moyennes des cotations observées sur la campagne 2010/2011 (soit, blé $=$ maïs $=214 € / t$, tourteau de soja $=327 € / \mathrm{t}$ ). Les prix des tourteaux de soja, colza et tournesol sont comparés dans la figure 4 . Ils montrent le lien fort existant entre les prix des tourteaux.

La présence simultanée de trois types de tourteaux de types 36, 32 et 29 a été étudiée pour déterminer les prix d'intérêt au-delà desquels l'incorporation de la matière première testée n'est pas possible car trop chère.

Ainsi, les prix d'intérêt des trois types de tourteaux exprimés par rapport au prix du tourteau de soja seraient tels que décrits dans le tableau 3.

Si on s'intéresse aux espèces potentiellement consommatrices, il apparaît que ce sont les volailles qui consommeraient quasi exclusivement le tourteau de type 36 alors que le tourteau 29 serait plutôt incorporé dans les formules bovines.

Cet intérêt particulier et nouveau du tourteau 36 pour les formules volailles pourrait être un atout comme source de protéines métropolitaines tracées et non OGM pouvant satisfaire aux exigences de cahiers des charges de production sous signe de qualité. Ainsi, le modèle précise que ce sont les pondeuses à plus de $50 \%$ qui utiliseraient le tourteau 36, suivies par les canards pour près de $40 \%$ et enfin les poulets label.

Quant au tournesol 29, il serait incorporé essentiellement dans les formules pour bovin viande à près de $70 \%$, puis par les lapins et les truies ce qui est caractéristique des utilisations du tourteau de tournesol non décortiqué plus riche en fibres et difficile à incorporer dans les régimes plus concentrés comme ceux de la volaille.

Les niveaux d'incorporation atteints dans les différentes formules ont également été étudiés et l'on distingue deux comportements différents pour les deux types de tourteaux.

Le tourteau 36 serait incorporé à des taux variant de 5 à $10 \%$ dans les formules pour volailles et pour réduire fortement la place du tourteau de soja dans les formules, il faudrait atteindre des taux d'incorporation bien supérieurs (de l'ordre de $30 \%$ et non réalisés en pratique) en formule canard par exemple et, soit accepter des surcoûts de formules, soit réduire assez sensiblement les ratios de prix par rapport au tourteau de soja. II faut cependant noter que ces résultats sont susceptibles de varier avec la conjoncture économique.

Le tourteau de tournesol 29 plus classique d'utilisation dans les formules moins concentrées en énergie et protéines attendraient des taux d'incorporation beaucoup plus élevés de l'ordre de

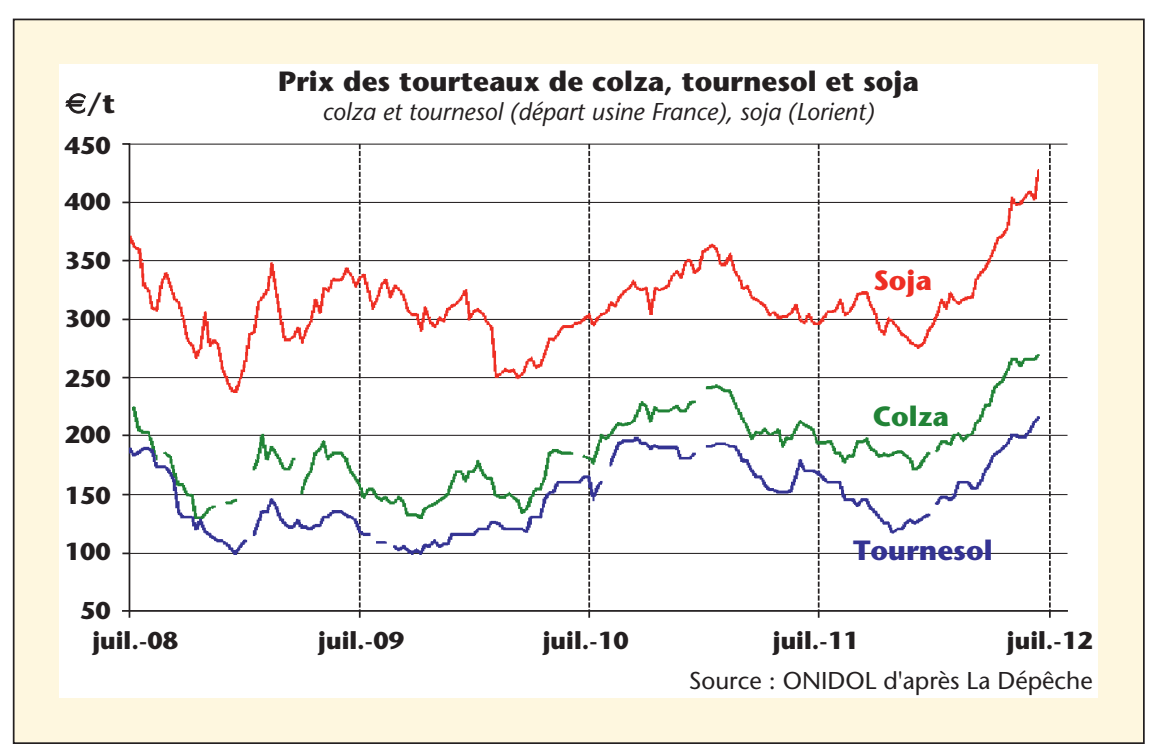

Figure 4. Comparaison des prix des tourteaux de soja, colza et tournesol. 
Tableau 3. Prix d'intérêt des trois types de tourteaux exprimés par rapport au prix du tourteau de soja.

\begin{tabular}{|ll|}
\hline $\begin{array}{l}\text { Type de tourteau } \\
\text { de tournesol }\end{array}$ & $\begin{array}{l}\text { Prix d'intérêt en rapport } \\
\text { au tourteau de soja }\end{array}$ \\
\hline 29 & 0,43 \\
\hline 32 & 0,50 \\
\hline 36 & 0,70 \\
\hline
\end{tabular}

$35 \%$ en bovin viande et $25 \%$ en lapin. De tels taux supposent une homogénéité de la qualité des tourteaux dans le temps pour minimiser les risques d'erreur sur les apports nutritionnels.

Enfin, l'augmentation des disponibilités en tourteau de tournesol 36 et 29 pourrait se traduire par une baisse des utilisations de tourteau de soja et de son mais aussi potentiellement de tourteau de colza assez proche des nouveaux tourteaux de tournesol.

\section{Conclusion}

La production de tourteau de tournesol de type 36 semble être une opportunité pour les productions de volailles telles que les pondeuses, les canards et les poulets labels qui, en plus de représenter une source métropolitaine de protéines végétales tracées pour l'alimentation animale, offrent au tournesol 36 les meilleurs prix d'intérêt avec des ratios de prix au tourteau de soja de l'ordre de $70 \%$ beaucoup plus intéressants que ceux du tourteau de tournesol non décortiqué de type 29 toujours inférieurs à $50 \%$. Par ailleurs, ces travaux montrent une certaine "spécialisation " des différents tourteaux métropolitains avec des débouchés relativement complémentaires. Ainsi le tourteau de tournesol 36 permettrait d'accéder au débouché volaille non encore atteint par le tourteau de colza et le tourteau de tournesol 29.
Cette complémentarité pourrait être un atout pour les différentes sources de protéines françaises qui seraient moins

Cependant, il convient de nuancer ces premiers résultats qui mériteraient un programme plus conséquent de travaux complémentaires allant des mesures de valeurs nutritionnelles de tourteaux issus de procédés de décorticage et trituration variés aux simulations économiques plus poussées.

Des mesures de valeurs nutritionnelles réalisées sur les différentes espèces animales et notamment sur les volailles sont indispensables. Des travaux ont ainsi été engagés par I'INRA de Nouzilly (37) et le Palmipôle (33) pour déterminer les valeurs nutritionnelles comparées de différentes qualités de tourteaux de tournesol (pailleux, semi décortiqué, décortiqué ou encore fermier gras) sur les canards en croissance et les coqs, poulets et dindonneaux. Des essais durant les phases de croissance des canards viennent compléter ces travaux.

Les études économiques sont également indispensables pour étudier en particulier l'impact des niveaux de prix du tourteau de soja (et de la robustesse des prix exprimés par rapport à ceux du tourteau de soja), des prix des 2 céréales dominantes que sont blé et maïs qui, par leur richesses différentes en acides aminés, influent sur les sources de concurrentes. protéines qui leur sont associées. Enfin, la présence sur le marché de produits d'importation tels que les tourteaux de tournesol ukrainiens de type 38 est également à étudier.

\section{Conflits d'intérêts : aucun}

\section{RÉFÉRENCES}

Beauguillaume A. Architecture des coques de tournesol. Oleoscope Bulletin du CETIOM $1982 ;(8): 20-1$

Carré P. Oilseed dehulling. Sustoil report, 2010, 31 p (www.creol.fr).

Dauguet S, Guillemain C, Carré P, Merrien A, Krouti M, Champolivier L. Effect of sunflower seeds quality on dehulling process in order to produce protein content guaranteed meal. Proc. $18^{\text {th }}$ Sunflower International Conference, Argentina, mars 2012.

INRA-AFZ. Tables de composition et de valeur nutritive des matières premières destinées aux animaux d'élevage. D. Sauvant, JM. Perez, G Tran (coord.), $2^{\mathrm{e}}$ édition revue et corrigée, INRA Editions, 2004.

Perez JM, Bourdon D, Baudet JJ, Evrard J. Prévision de la valeur énergétique des tourteaux de tournesol à partir de leurs teneurs en constituants pariétaux. Journées de la recherche porcine 1986 ; 18 : 35-46.

Sket-Cimbria. Dehulling of oilseeds. SKET's new electrical dehulling process is employed for large-scale processing of sunflower seed. Oils and Fat International $1991 ; 7$ : 44-7.

Tostain S, Chervier P, Laulan A, Kermorgant T. Amélioration de l'autonomie énergétique et de l'impact environnemental d'une unité de trituration de tournesol par l'implantation conjointe d'un atelier de décorticage et d'une chaudière à coques. OCL $2012 ; 19$ (in press).

Villamide MJ, San Juan LD. Effect of chemical composition of sunflower seed meal on its true metabolizable energy and amino acid digestibility. Poultry Science 1998 ; 77 : 1884-92. 